\title{
Early Gastric Cancer with Mixed-Type Histology: A Mixed Bag or Pure Evil?
}

\author{
Mohamed O. Othman ${ }^{1}$
}

Published online: 15 November 2019

(c) Springer Science+Business Media, LLC, part of Springer Nature 2019

Undifferentiated or poorly differentiated gastric adenocarcinoma carries a higher risk of lymph node metastasis (LNM) than do more well-differentiated tumors, which was shown to be as high as $9 \%$ in a published trial [1]. In a meta-analysis of 23 studies focusing on LNM in undifferentiated gastric adenocarcinoma, the following risk factors were identified as predictors for LNM: female sex, age $>60$ years, a tumor $>2 \mathrm{~cm}$, ulcerated lesions, submucosal invasion, lymphovascular invasion, non-signet ring carcinoma, or tumor located outside the gastric corpus [2]. Although the risk of LNM for undifferentiated early gastric adenocarcinoma (EGC) is well studied, tumors with mixed differentiation types are not well studied. The Japanese classification of gastric carcinoma does not recognize mixed differentiation as a separate entity. A tumor with mixed differentiation where the majority of the tumor is well-differentiated is considered as a differentiated tumor despite the presence of a small, undifferentiated component. The same holds true for a predominantly undifferentiated tumor with a small differentiated component that is treated as an undifferentiated tumor [3]. Whether a tumor with mixed differentiation is truly a different entity and whether it carries the same risk of LNM as seen in an undifferentiated tumor is a matter of debate. In this issue of Digestive Disease and Sciences, Bang et al. [4] tackled the difficult task of assessing the risk of LNM and outcomes of endoscopic submucosal dissection (ESD) in patients with EGC of mixed-type histology (MH). The systematic review included eight retrospective studies that reported the outcomes of ESD in the management of EGC in 458 patients with MH. The authors found that EGC with MH (EGC-MH) has higher rates of submucosal invasion compared with purely differentiated or even purely

Mohamed O. Othman

mohamed.othman@bcm.edu

1 Division of Gastroenterology, Baylor College of Medicine, 7200 Cambridge St., 8th Floor, Suite 8B, Houston, TX 77030, USA undifferentiated EGC. One of the included trials showed a higher rate of lymphovascular invasion in EGC with $\mathrm{MH}$ compared to EGC with purely undifferentiated histology [5]. Pooled meta-analysis showed an en-bloc resection rate of $94.6 \%$ with a curative resection rate of only $55.1 \%$ (95\% CI 50.4-59.6\%) for EGC-MH; significantly less than reported for other types of EGC. Finally, one of the included studies in this systematic review showed a higher recurrence rate after ESD in EGC-MH compared with purely undifferentiated gastric cancer [6]. I congratulate the authors on their extensive research and analysis of the literature. Data regarding EGC-MH are extremely rare; the authors had to 'read between the lines' to extract such valuable information. The take-home point from this study is that EGC-MH should be treated with extreme caution given its high rate of non-curative resection in addition to a higher recurrence rate after endoscopic resection. The presence of a differentiated component within these tumors did not offer any advantage compared to the purely undifferentiated gastric cancer. As was alluded to some of the included studies, EGC with mixed histology can behave even more aggressively than do undifferentiated tumors. Recent data suggest that there is a subset of patients with undifferentiated EGC who are at low risk of LNM. In a retrospective study of 1425 patients with undifferentiated gastric cancer treated surgically, no LNM was detected in patients with an intramucosal tumor $<4 \mathrm{~cm}$ with no ulceration or evidence of lymphovascular invasion in the resected specimen (95\% CI 0-6\%) [7].

With regard to the lower curative resection rates in EGC-MH, it seems that the lateral margin positivity was the most common reason for non-curative resection in two of the included studies. This suggests that since EGC-MH has ill-defined margins, a wider resection should be recommended. For this reason, it is always advisable to biopsy the suspected gastric cancer followed by a biopsy of the surrounding mucosa in a separate container in order to assess for higher risk mucosal pathology such as atrophic gastritis, intestinal metaplasia, or dysplasia. 
The data generated from this systematic review are in line with the growing body of the literature on the behavior of EGC-MH. Choi et al. in a study of the clinicopathological features of 2665 surgically resected EGC divided the sample into 3 groups based on the Lauren classification: intestinal type (differentiated tumors), diffuse type (undifferentiated tumors), and mixed type (mixed intestinal and diffuse type). The authors found that Lauren's mixed-type EGC had a significantly higher rate of LNM compared with both Lauren's intestinal and diffuse type $(22.8 \%$ vs. $8.2 \%$ and $9.1 \%$, respectively) [8]. Similar findings were noted in another retrospective study by Pyo et al. [9] which assessed risk factors for LNM in patients with mixed-type Lauren classification. LNM was seen in $15.4 \%$ of 336 patients with Lauren's mixed-type EGC.

In conclusion, EGC-MH should be regarded as an entity apart from other types of GC. The presence of differentiated components in these tumors does not connote a benign course. In fact, these tumors could be more aggressive or at minimum as aggressive as purely undifferentiated tumors and should be treated as such.

\section{Compliance with Ethical Standards}

Conflict of interest Consultant for Boston Scientific, Olympus, Lumendi.

\section{References}

1. Fang WL, Huang KH, Lan YT, et al. The risk factors of lymph node metastasis in early gastric cancer. Pathol Oncol Res. 2015;21:941-946.
2. Zhao X, Cai A, Xi H, et al. Predictive factors for lymph node metastasis in undifferentiated early gastric cancer: a systematic review and meta-analysis. J Gastrointest Surg. 2017;21:700-711.

3. Japanese Gastric Cancer A. Japanese gastric cancer treatment guidelines 2014 (ver. 4). Gastric Cancer. 2017;20:1-19.

4. Bang CS, Yang YJ, Lee JJ, et al. Endoscopic submucosal dissection of early gastric cancer with mixed-type histology: a systematic review. Dig Dis Sci. (Epub ahead of print). https://doi. org/10.1007/s10620-019-05761-w.

5. Lee JH, Kim JH, Rhee K, et al. Undifferentiated early gastric cancer diagnosed as differentiated histology based on forceps biopsy. Pathol Res Pract. 2013;209:314-318.

6. Han JP, Hong SJ, Kim HK. Long-term outcomes of early gastric cancer diagnosed as mixed adenocarcinoma after endoscopic submucosal dissection. J Gastroenterol Hepatol. 2015;30:316-320.

7. Horiuchi Y, Ida S, Yamamoto N, et al. Feasibility of further expansion of the indications for endoscopic submucosal dissection in undifferentiated-type early gastric cancer. Gastric Cancer. 2019. https://doi.org/10.1007/s10120-019-01003-0.

8. Choi J, Chung H, Kim J, Kim JL, Kim SG, Jung HC. Determining the current indications for endoscopic submucosal dissection in patients with Lauren mixed-type early gastric cancer. J Gastroenterol Hepatol. 2019. https://doi.org/10.1111/jgh.14846.

9. Pyo JH, Lee H, Min BH, et al. Early gastric cancer with a mixedtype Lauren classification is more aggressive and exhibits greater lymph node metastasis. J Gastroenterol. 2017;52:594-601.

Publisher's Note Springer Nature remains neutral with regard to jurisdictional claims in published maps and institutional affiliations. 